\title{
Quantitative Characteristics of Neutrophil Extracellular Traps in Tumor Tissues in Colorectal Cancer
}

\author{
Larissa Muravlyova ${ }^{1}$, Mereke Maratkyzy ${ }^{2 \star}$, Nailya Kabildina ${ }^{2}$, Ksenia Mkhitaryan $^{3}$, Olga Ponamareva ${ }^{1}$, Ryszhan Bakirova ${ }^{4}$, \\ Vilen Molotov-Luchanskiy ${ }^{4}$, Valentina Sirota ${ }^{2}$ \\ ${ }^{1}$ Department of Biological Chemistry, Karaganda Medical University, Karaganda, Kazakhstan; ${ }^{2}$ Department of Oncology and \\ Radiation Diagnostics, Karaganda Medical University, Karaganda, Kazakhstan; ${ }^{3}$ Department of Informatics and Biostatistics, \\ Karaganda Medical University, Karaganda, Kazakhstan; ${ }^{4}$ Department of Internal Diseases, Karaganda Medical University, \\ Karaganda, Kazakhstan
}

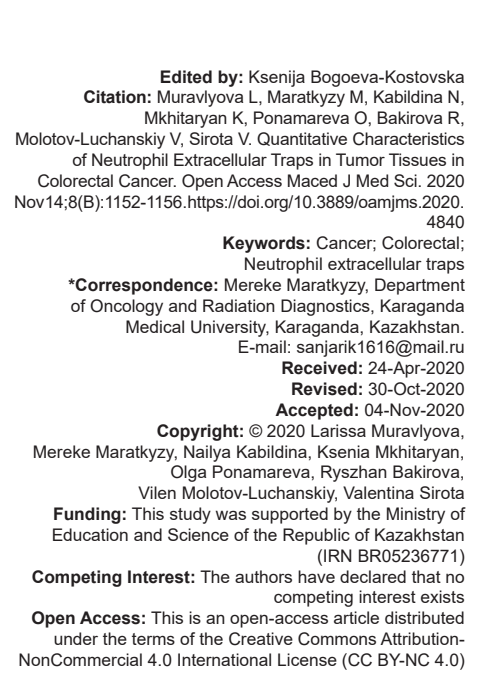

Abstract

BACKGROUND: Recently, the role of neutrophil extracellular traps (NETs) in oncopathology, including colorectal cance (CRC), has become a subject of discussion. Literature review showed that in CRC, there are separate experimental and clinical studies devoted to assess the ability of neutrophils to form NET. A methodological approach, common to these studies, was that the ability of blood neutrophils in patients with CRC to form NET was evaluated in vitro.

AIM: We undertook a study of the NETs presence in the tumor tissues, on the periphery of the tumor, and in healthy tissues with $\mathrm{CRC}$ at different stages and different types of therapy.

METHODS: The study was carried out in the framework of the scientific and technical program "Personalized approach to the management of number of significant diseases" developed by the Non-commercial Joint-Stock Company "Karaganda Medical University," funded by the Ministry of Education and Science of the Republic of Kazakhstan

RESULTS: Patients with CRC II and III stages are more likely to have NET in the center, on the periphery of the tumo and in healthy tissues adjacent to the tumor than in patients with CRC of Stage I. It was also found that radiation therapy as a pre-operative preparation contributed to an increase in the number of NET in the center of the tumor in patients with Stage I rectal cancer

CONCLUSION: Thus, our data revealed an increase in NET in the center and periphery of CRC, and an increase in their number after pre-operative radiation therapy was shown. The next stage of our study will be devoted to study the structural features of NET and their interaction with other effector cells and tumor cells.

\section{Introduction}

Colorectal cancer $(\mathrm{CRC})$ is one of the most common malignant tumors in the world, the incidence is growing in both developed and developing countries. It takes the third place in the general structure of malignant tumors and is the fourth leading cause of cancer mortality worldwide [1].

In Kazakhstan, in recent years, there has been an increase in the incidence of CRC. The intensive indicator of CRC incidence in 2018 amounted to $9.1 \%$ per 100 thousand people $(2017-9.4 \%)$ with an increase rate of $3.7 \%$, which in absolute numbers are 1667 new cases per year. The intensive indicator of CRC incidence in 2018 amounted to $8.4 \%$ per 100 thousand people $(2017-8.1 \%)$ with a growth rate $4.6 \%$, which in absolute numbers are 1551 new cases per year [2].

$\mathrm{CRC}$ takes the fifth place in cancer mortality rate in Kazakhstan, after lung cancer, stomach cancer, breast cancer, and pancreatic cancer (5.3\%). Rectal cancer takes the seventh place in cancer mortality rate (4.8\%) [2]

The incidence and urgency problem of CRC requires an in-depth study of the molecular-cellular changes accompanying with disease development and treatment.

The leading role in the immune system response is played by neutrophils, which have high cytotoxic potential and intertissue mobility. The cytotoxic potential of neutrophils is realized through various mechanisms, including through the formation of neutrophil extracellular traps (NETs). Different mechanisms of NET formation have been described: Suicidal NETosis and vital (intravital) NETosis. Suicidal NETosis is induced by various chemical factors, the interaction of which with receptors on the neutrophil membrane triggers a cascade of intracellular transformations resulting in the destruction of granules, chromatin decondensation in the nucleus, nuclear membrane decay, attachment of granular proteins to nuclear fibrils, rupture of the cell membrane, 
and the release of a complex of DNA and histones into the environment with the formation of a network. Granular proteins are represented by myeloperoxidase, elastase, lysozyme $\mathrm{C}$, etc., which determine the efficiency of pathogenic microorganisms degradation [3].

Vital NETosis is induced by bacterial factors; it is not accompanied by cell lysis; the nucleus DNA is released using vesicles [4].

Recently, the role of NET in oncopathology, including $\mathrm{CRC}$, has become a subject of discussion. Literature review showed that in $\mathrm{CRC}$, there are separate experimental and clinical studies devoted to assess the ability of neutrophils to form NET. A methodological approach, common to these studies, was that the ability of blood neutrophils in patients with CRC to form NET was evaluated in vitro. After isolation, neutrophils were primed with various stimuli and their ability to form NET relative to those of the control group was evaluated. Based on these studies, it was concluded that an increase in the number of neutrophils forming NET contributes to increased metastasis of CRC [5], [6].

Literature review showed different opinions on the role of NET in CRC. In this regard, we undertook a study of the NETs presence in the tumor tissues, on the periphery of the tumor, and in healthy tissues with CRC at different stages and different types of therapy.

\section{Materials and Methods}

\section{Study design}

This was a clinical, prospective study.

\section{Study condition}

The study was carried out in the framework of the scientific and technical program STP 0.0821 "Personified Approach to the Management of a Number of Significant Diseases" funded by the Ministry of Education and Science of the Republic of Kazakhstan (IRN BR05236771).

\section{method}

\section{Sample size justification and recruitment}

The study involved 168 patients with an established diagnosis of CRC. These are all patients treated at the Regional Oncological Dispensary of Karaganda for 2018-2019. All patients were divided into seven age categories: Up to 30 years old, 31-40 years old, 41-50 years old, 51-60 years old, 61-70 years old, 71-80 years old, and over 80 years old. Inclusion in the study meant familiarizing the patient with the essence of the study and signing an informed consent.

\section{Ethical approval}

The study was approved by the Bioethics Committee of Karaganda Medical University.

The clinical diagnosis was established according to ICD 10 [7]; for cancer stages, classification of the TNM classification developed by the International Cancer Union, 2019 version, was used [8].

\section{Study description}

On admission to the hospital, all examined patients underwent a general clinical examination at the outpatient stage. Morphological verification of tumors was carried out in the cytological and morphological laboratories of the Regional Clinical Oncology Center, Karaganda. All patients have histological verification, mainly, adenocarcinoma with localization of the tumor in the colon and rectosigmoid and ampullary sections of the rectum, with localization of the tumor in the perineal region - squamous cell carcinoma.

After the diagnosis verification and a full examination, all patients underwent surgical treatment.

Out of 168 patients with colon and rectum cancer, 82 patients were examined to NETs.

The number of extracellular DNA networks in tumor imprint smears was estimated during the operation. A imprint smear was taken from the center of the tumor dissected in the sector, on the periphery of the tumor and in healthy tissues on glass slides and stained according to azure-eosin according to the May-Grunwald method [9], with a light microscope at 1400 times magnification with extracellular DNA counting by the method described by Dolgushin et al. [9], [10], [11]. Counting was carried out on 10 fields of view. NET photographs were obtained using the ToupView 3.7 program.

For the statistical processing of the obtained results, the mathematical statistics procedures implemented in the STATISTICA 10.0 and EXCEL applications were used. Preliminarily obtained results were checked for normal distribution. Results are presented medians and quartiles, the difference between the indicators in the compared groups was calculated according to Mann-Whitney.

Patients' characteristics: Out of 82 patients, there were 30 women and 52 men aged 37-85, the average age of the patients was $65.5 \pm 8.4$. There were 14 $(17.0 \%)$ patients with the first stage of cancer, 49 (59.8\%) people with the second stage, and $19(23.2 \%)$ patients with the third stage of cancer. CRC was diagnosed in 43 $(52.4 \%)$ patients, of which $33(76.7 \%)$ patients received a neoadjuvant course of radiation therapy. In terms of combined treatment, at the first stage, they conducted a neoadjuvant course of remote gamma therapy on the ROCUS AM apparatus in the rectal region, pararectal tissue, and regional lymph nodes (single boost dose of 20 Gy and total boost dose of $40 \mathrm{~Gy}$ ). 
Colon cancer was diagnosed in 39 (47.6\%) patients, of which 19 (48.7\%) patients were diagnosed with sigmoid colon cancer. All patients underwent surgical treatment.

All patients were divided into two groups. The first group included patients who had undergone surgical treatment immediately after the diagnosis of CRC. This group included 10 patients with Stage I CRC, 28 patients with Stage II CRC, and 11 patients with Stage III CRC. The second group included patients who had received a neoadjuvant course of radiation therapy.

This group included 4 patients with Stage I rectal cancer, 21 patients with Stage II rectal cancer, and 8 patients with Stage III rectal cancer.

\section{Results}

The research results are presented in Table 1.

Table 1: Quantitative characteristics of tissue NETs in patients with CRC (median/25-57\% Cl)

\begin{tabular}{|c|c|c|c|}
\hline Cancer prevalence & $\begin{array}{l}\text { Colorectal } \\
\text { cancer patients } \\
\text { after surgery }\end{array}$ & $\begin{array}{l}\text { Colorectal cancer patients } \\
\text { after neoadjuvant radiation } \\
\text { therapy and surgery }\end{array}$ & $p \leq 0.05$ \\
\hline \multicolumn{4}{|l|}{ I stage } \\
\hline At the center of the tumor & $1.5(0 ; 15)$ & $7.5(1 ; 21,5)$ & $p \geq 0.05$ \\
\hline At the periphery of the tumor & $11(0 ; 24)$ & $8(3 ; 18)$ & $p \leq 0.05$ \\
\hline In healthy tissues & $0(0 ; 0)$ & $2,5(0 ; 9)$ & \\
\hline \multicolumn{4}{|l|}{ II stage } \\
\hline At the center of the tumor & $8(0 ; 16)$ & $10(5 ; 15)$ & $p \geq 0.05$ \\
\hline At the periphery of the tumor & $11(4 ; 22)$ & $11(4 ; 25)$ & $p \geq 0.05$ \\
\hline In healthy tissues & $0(0 ; 3)$ & $0(0 ; 0)$ & \\
\hline \multicolumn{4}{|l|}{ III stage } \\
\hline At the center of the tumor & $5(0 ; 11)$ & $12(0 ; 30)$ & $p \geq 0.05$ \\
\hline At the periphery of the tumor & $7.5(0,5 ; 13)$ & $8(1 ; 19)$ & $p \geq 0.05$ \\
\hline In healthy tissues & $0(0 ; 9.5)$ & $0(0 ; 25)$ & \\
\hline
\end{tabular}

As a study result, it was found that at patients with Stage I CRC included in Group 1, after surgery, the number of NET varied. Hence, in the center of the tumor, NET was found in $50 \%$ of patients with CRC; the median value was $-1.5(0 ; 15)$. At the periphery of the tumor, NET was found in $70 \%$ of patients in this group; the median value was $11(0 ; 24)$. In healthy tissue adjacent to the tumor, NET was found in $10 \%$ of patients; the median value was $0(0 ; 0)$.

In patients with Stage II CRC, included in Group I, after surgical treatment, NET in the center of the tumor was found in $72 \%$ of patients; the median value was $8(0 ; 16)$ (Figure 1). At the periphery of the tumor, NET was found in $89 \%$ of patients in this group, the median value was $11(4 ; 22)$. In healthy tissue adjacent to the tumor, NET was found in $25 \%$ of patients; the median value was $0(0 ; 3)$.

In patients with Stage III CRC, included in Group 1, after surgical treatment, NET in the center of the tumor was found in $63 \%$ of patients; the median value was $5(0 ; 11)$. At the periphery of the tumor, NET was found in $72 \%$ of patients in this group, the median value was $7.5(0.5 ; 13)$ (Figure 2$)$. In healthy tissue adjacent to the tumor, NET was found in $27 \%$ of patients; the median value was $0(0 ; 9.5)$.

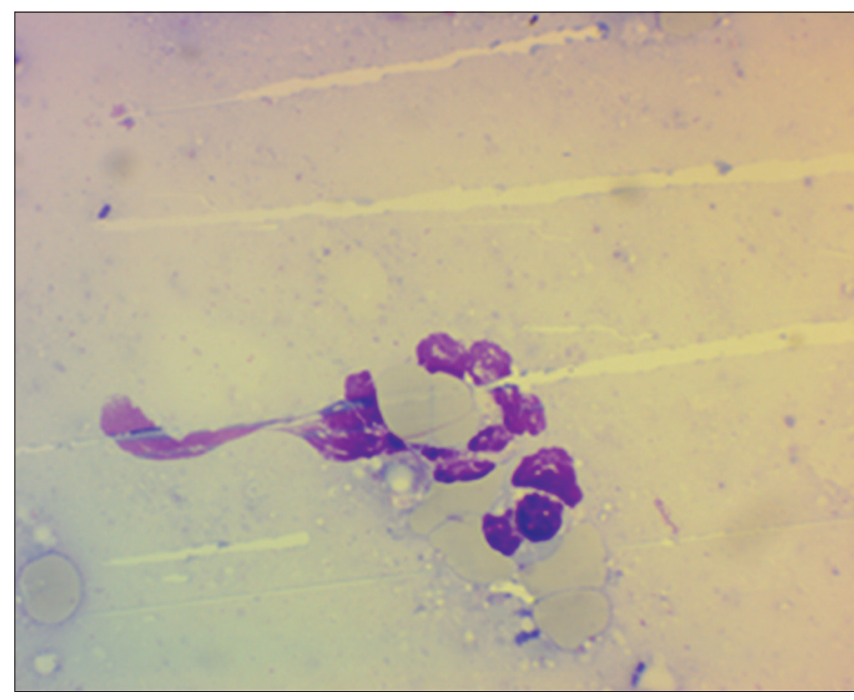

Figure 1: Neutrophil extracellular traps in the tumor center of a patient with colorectal cancer (May-Grunwald azure-eosin staining, 1400x)

Neoadjuvant preparation, then surgery, NET in the center of the tumor was found in $75 \%$ of patients; the median value was $7.5(1 ; 21.5)$. At the periphery of the tumor, NET was found in $100 \%$ of patients in this group; the median value was $8(3 ; 18)$. In healthy tissues adjacent to the tumor, GNI was found in $50 \%$ of patients in this group; the median value was $2.5(0 ; 9)$.

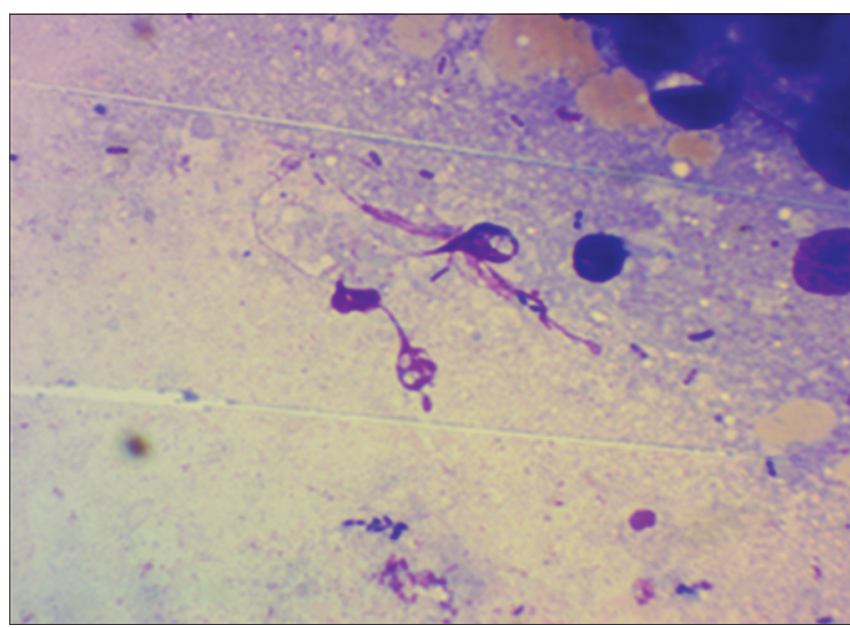

Figure 2: Neutrophil extracellular traps in the tumor periphery of a patient with colorectal cancer (May-Grunwald staining with azureeosin, 1400x)

In patients with Stage II CRC who received radiation therapy as a neoadjuvant preparation, then surgery, NET in the center of the tumor was found in $81 \%$ of patients, the median value was $10(5 ; 15)$ (Figure 3). At the periphery of the tumor, NET was found in $76 \%$ of patients, the median value was $11(4 ; 25)$. In normal tissue, NET was found in $24 \%$ of the examined patients, the median value was $0(0 ; 0)$.

In patients with Stage III CRC who received radiation therapy as a neoadjuvant preparation, then surgery, NET in the center of the tumor was found in $75 \%$ of patients, the median value was $12(0 ; 30)$ 
(Figure 4). At the periphery of the tumor, NET was found in $87.5 \%$ of the examined patients; the median value was $8(1 ; 19)$. In normal tissue, NET was found in $50 \%$ of the examined patients; the median value is $0(0 ; 25)$.

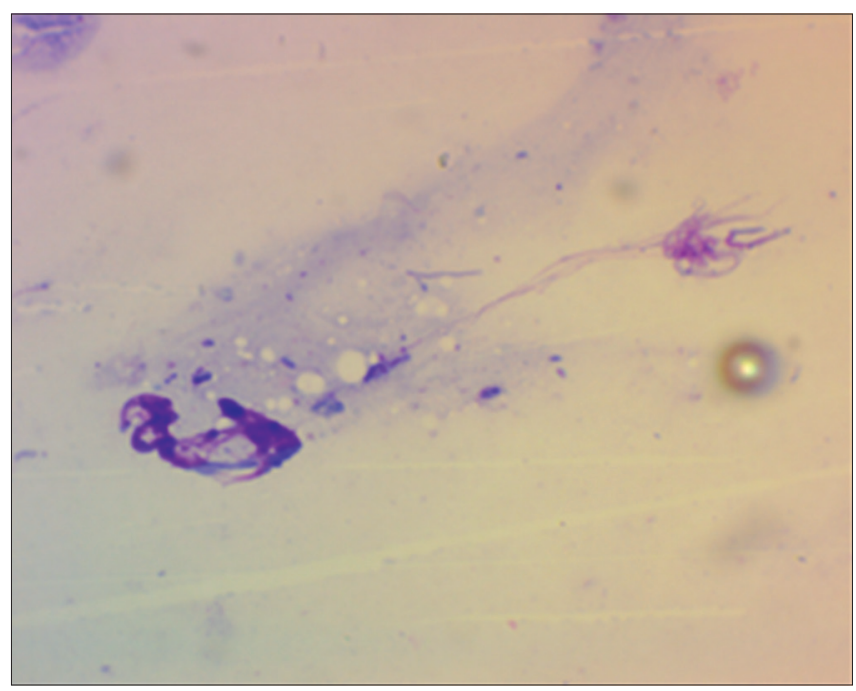

Figure 3: Neutrophil extracellular traps in the tumor center of a patient with colorectal cancer after radiation therapy (May-Grunwald azureeosin staining, 1400x)

\section{Discussion}

As a study result, it was found that in patients with $\mathrm{CRC}$ in the center of the tumor and on the periphery of the tumor, NET is detected. Our data supplement the results of Arelaki et al. [12], which showed the presence of NET in tumor sections of CRC. We did not observe a decrease in the amount of NET from the center to the periphery of the tumor. As our studies showed, the amount of NET in the center of the tumor and on the periphery was comparable.

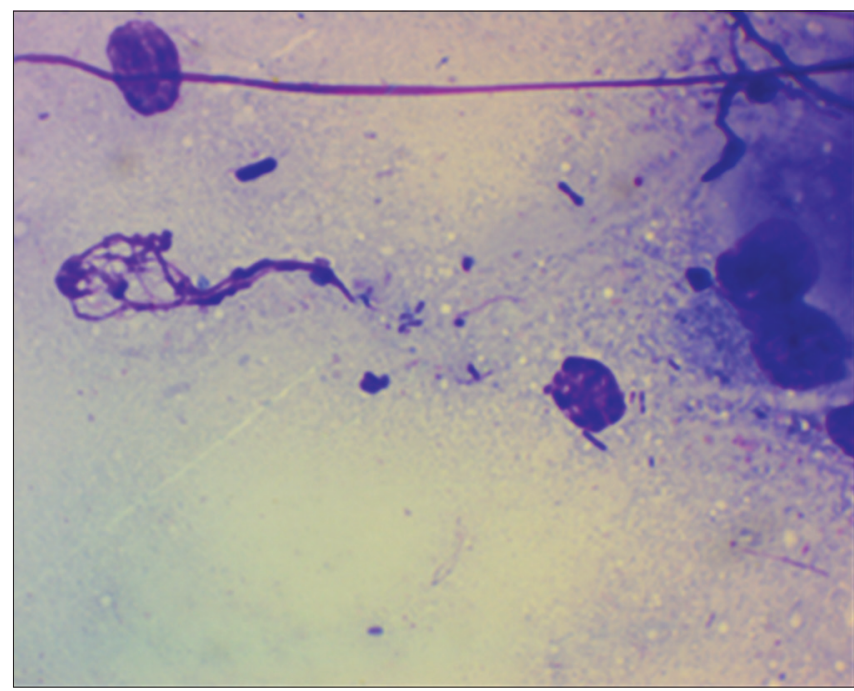

Figure 4: Neutrophil extracellular traps in the tumor periphery of a patient with colorectal cancer after radiation therapy (May-Grunwald azure-eosin staining, 1400x)
Patients with CRC of Stages II and III are more likely to have NET in the center, on the periphery of the tumor and in healthy tissues adjacent to the tumor than in patients with CRC of Stage I. It was also found that radiation therapy as a pre-operative preparation contributed to an increase in the number of NET in the center of the tumor in patients with Stage I rectal cancer.

\section{Conclusion}

As follows from the data obtained, we revealed an increase in NET in the center and periphery of CRC, and an increase in their number after pre-operative radiation therapy was shown. Thus, it is of interest to evaluate the ability of neutrophils to form extracellular traps in case of socially significant diseases. The next stage of our study will be devoted to study the NET structural features and their interaction with other effector cells and tumor cells.

\section{Data availability}

The quantitative data used to support the findings of this study are available from the corresponding author on request.

\section{References}

1. Bray F, Ferlay J, Soerjomataram I, Siegel RL, Torre LA, Jemal A. A global cancer statistics 2018: GLOBOCAN estimates of incidence and mortality worldwide for 36 cancers in 185 countries. CA Cancer J Clin. 2018;68(6):394-424. https://doi. org/10.3322/caac.21492

\section{PMid:30207593}

2. Republic of Kazakhstan. Indicators of the Oncological Service of the Republic of Kazakhstan for 2018 (Statistical and Analytical Materials). Almaty: Republic of Kazakhstan; 2019. p. 210.

3. Brinkmann V. Neutrophil extracellular traps in the second decade. J Innate Immun. 2018;10(5-6):414-21. https://doi. org/10.1159/000489829

PMid:29909412

4. Yipp BG, Kubes P. NETosis: How vital is it? Blood. 2013;122(16): 2784-94. https://doi.org/10.1182/blood-2013-04-457671 PMid:24009232

5. Al-Haidari AA, Algethami N, Lepsenyi M, Rahman M, Syk I, Thorlacius $\mathrm{H}$. Neutrophil extracellular traps promote peritoneal metastasis of colon cancer cells. Oncotarget. 2019;10(12):123849. https://doi.org/10.18632/oncotarget.26664 PMid:30815227

6. Richardson JJ, Hendrickse C, Gao-Smith F, Thickett DR Neutrophil extracellular trap production in patients with colorectal cancer in vitro. Int J Inflam. 20172017:4915062. https://doi.org/10.1155/2017/4915062 
PMid:28828191

7. International Statistical Classification of Diseases and Related Health Problems, $10^{\text {th }}$ Revision. Available from: https://www. mkb-10.com. [Last accessed on 2020 Oct 13].

8. Sobin LH, Gospodarowicz MK, Wittekind C, International Union Against Cancer (UICC). TNM Classification of Malignant Tumours. $7^{\text {th }}$ ed. New York: Wiley-Blackwell; 2019.

9. Available from: http://www.medlz.ru/jdo-335.html. [Last accessed on 2020 Oct 13].

10. Dolgushin II, Andreeva YS. Method for de Tection of Extracellular
Neutrophilic Traps: Russian Federation Patent for Invention, No. 2384844; 2008.

11. Dolgushin J. Andreeva S, Savochkina AJ. Neytrofil'nyye Vnekletochnyye Lovushki i Metody Otsenki Funktsional'nogo Statusa Neytrofilov. Moscow: RAMS Publishing House; 2009.

12. Arelaki S, Arampatzioglou A, Kambas K, Papagoras C, Miltiades P, Angelidou I, et al. Gradient infiltration of neutrophil extracellular traps in colon cancer and evidence for their involvement in tumour growth. PLoS One. 2016;11(5):e0154484. https://doi.org/10.1371/journal.pone.0154484

PMid:27136460 